\title{
Overexpression of flotillin-1 is involved in proliferation and recurrence of bladder transitional cell carcinoma
}

\author{
YAWEI GUAN ${ }^{1}$, HAIYAN SONG ${ }^{2}$, GUOHUI ZHANG ${ }^{1}$ and XING AI ${ }^{1}$ \\ ${ }^{1}$ Department of Urinary Surgery, Beijing Military Region General Hospital, Beijing 100700; \\ ${ }^{2}$ Department of Blood Transfusion, The 309th Hospital of Chinese PLA, Beijing 100091, P.R. China
}

Received March 19, 2014; Accepted May 2, 2014

DOI: 10.3892/or.2014.3221

\begin{abstract}
Flotillin-1 (FLOT1) is known to have a role in tumorigenesis; however, the effect of FLOT1 on proliferation and recurrence of human transitional cell carcinoma (TCC) is unclear. Samples from 156 TCC patients and 142 patients undergoing open bladder surgery for indications other than TCC were used in the present study. FLOT1 protein expression was determined by immunohistochemistry and western blot analysis, and mRNA expression was detected by RT-PCR and real-time PCR. A FLOT1-expressing pcDEF3 vector was stably transfected into 4 TCC cell lines and FLOT1 expression was decreased by RNAi. Proliferative analysis of TCC cells was detected by the WST-1 assay and a xenograft model using BALB/C nude mice. The association between FLOT1 expression and TCC recurrence was also analyzed by adhesion, migration and invasion assays. FLOT1 expression in TCC was significantly overexpressed compared to normal urothelial tissue, and the level of FLOT1 expression was significantly correlated with tumor size, pathologic grade, clinical stage and recurrence. In addition, FLOT1 significantly increased the proliferative ability of TCC cells in vitro and in vivo. TCC cells with a high level of FLOT1 expression exhibited a higher level of adhesion, migration and invasion. FLOT1 expression was shown to be upregulated in human TCC. These findings suggest that FLOT1 plays an important role in the proliferation and recurrence of TCC and that silencing FLOT1 expression might be a novel therapeutic strategy.
\end{abstract}

\section{Introduction}

Bladder carcinoma is a common malignancy worldwide, and is associated with a high morbidity and mortality (1). Approximately $90 \%$ of bladder carcinomas are transitional cell carcinomas (TCCs); 70-80\% of TCCs are non-muscle-

Correspondence to: Dr Yawei Guan, Department of Urinary Surgery, Beijing Military Region General Hospital, 2 Chaoyangmen Beixiao Street, Dong Cheng, Beijing 100700, P.R. China E-mail: yaweiguan@sina.com

Key words: flotillin-1, transitional cell carcinoma, bladder, therapeutic target, tumorigenesis, recurrence invasive (pTa-pT1) and 20-30\% of TCCs are muscle-invasive (pT2-pT4). Moreover, 50-70\% of these non-muscle-invasive TCCs will recur and 10-30\% will become muscle-invasive TCCs (2). The prognosis of TCCs mainly depends on the clinical stage and histologic grade of the tumor at the time of diagnosis (3). The 5-year overall survival rate for non-muscleinvasive TCCs is $90 \%$, and 60, 35 and $25 \%$ for pT2, pT3 and pT4 TCCs, respectively $(4,5)$. Despite the advances in treatment and surveillance strategies, the mortality rate amongst patients with TCCs is on the rise, as is the tumor recurrence rate. Thus, the mechanisms underlying TCC recurrence also require elucidation, and studies seeking to further understand TCC recurrences are necessary for the development of novel approaches and improved therapeutic strategies.

The dynamic structure of the cell membrane usually plays a vital role in some biological processes, such as cell growth, survival and metastasis. Lipid rafts have been reported to be associated with the development of a number of malignancies $(6,7)$ and serve as a physical platform on which molecules coordinate signaling pathways (8-10). Therefore, it has been demonstrated that lipid rafts may be a distinctive therapeutic target and treatment strategy to overcome malignant cancers $(11,12)$. Flotillin-1 (FLOT1) and FLOT2 are homologous isoforms of the flotillin protein family and are essential markers of lipid rafts (13-15). FLOT1 and FLOT2 interact with each other to form a complex and have an important role in biological processes, such as membrane receptor signaling, membrane trafficking, cell adhesion and invasion (16). Increased activation of Fyn kinase leads to the translocation of FLOT1 and FLOT2 from the membrane to intracellular organelles, and participates in tyrosine kinaseregulated endocytic processes (17). FLOT1 and FLOT2 have been reported to activate signaling pathways via binding to membrane receptor kinases; for example, stimulation with insulin can prompt the complex formation of Cbl-CAP and FLOT1, which launches signal conduction that is important for the uptake of glucose in adipocytes (18). Previous studies have indicated that FLOT1 and FLOT2 are also involved in tumorigenesis and progression of human carcinomas $(19,20)$. FLOT1 expression has been reported to be upregulated in carcinomas of epithelial origin, such as colorectal carcinomas and esophageal squamous cell carcinomas $(21,22)$. Moreover, overexpression of FLOT1 enhances the activity of Aurora B kinase and leads to incorrect attachment of microtubules to 
kinetochores, suggesting that FLOT1 can cause genomic instability and may be associated with tumorigenesis (23). In another study, overexpression of FLOT1 was also observed and prompted proliferation of prostate cancer cells (24). These findings suggest that FLOT1 may play an important role in the progression and development of malignant carcinomas; however, the expression of FLOT1 has not been reported and the role of FLOT1 in human TCC remains unclear.

In the present study, we determined the expression of FLOT1 and its effect on the proliferation and recurrence of human TCC. Our results suggest that FLOT1 expression is upregulated in TCC and that FLOT1 can enhance the proliferation of TCC cells in vitro and in vivo. Thus, FLOT1 may serve as a therapeutic target for TCC. Moreover, FLOT1 increased the adhesion, migration and invasion of TCC cells, suggesting that FLOT1 may be involved in TCC recurrence and that FLOT1 may be a candidate marker for predicting TCC recurrence.

\section{Materials and methods}

Patients and samples. The study included 156 TCC patients treated in the Department of Urology of Beijing Military Region General Hospital between 2001 and 2011. No patients received radiotherapy or chemotherapy before surgery. The histologic cell type of the bladder cancer samples was evaluated by an experienced pathologist without prior knowledge of the patient disease data; all tumors in the present study were conventional TCC. Normal urothelial tissues were also obtained from 142 patients undergoing open bladder surgery for indications other than TCC, such as benign prostatic hyperplasia and trauma. Tumor stage was classified according to the UICC 2009 TNM classification system, and the histologic grade was assessed according to the WHO 2004 grading system of TCC. All TCC samples and normal bladder tissues were obtained, formalinfixed and paraffin-embedded. In addition, all samples in the present study were also frozen in liquid nitrogen immediately after surgical resection, and kept at $-90^{\circ} \mathrm{C}$ until total RNA and protein extraction. The study was approved by the Ethics Committee of the Beijing Military Region General Hospital.

Cell culture. Four TCC cell lines (RT112, 253J, T24 and TCCSUP) were purchased from the American Type Culture Collection (ATCC; Manassas, VA, USA) and cultured in complete medium, consisting of RPMI-1640 (Gibco Bio-Cult, Glasgow, Scotland), supplemented with $25 \mathrm{mM}$ HEPES, $2 \mathrm{mM}$ L-glutamine, $100 \mathrm{U} / \mathrm{ml}$ of penicillin or $100 \mu \mathrm{g} / \mathrm{ml}$ of streptomycin, $10 \%$ heat-inactivated fetal bovine serum (FBS), and $1 \%$ non-essential amino acids. All TCC cell lines were maintained as monolayers in $10-\mathrm{cm}$ plastic dishes and cultured in a humidified atmosphere containing $5 \% \mathrm{CO}_{2}$ at $37^{\circ} \mathrm{C}$.

Immunohistochemistry. Paraffin sections $(4 \mu \mathrm{m})$ were deparaffinized in xylene and rehydrated with graded alcohol. Endogenous peroxidase activity was blocked with $0.3 \%$ hydrogen peroxide for $20 \mathrm{~min}$. All sections were also blocked with $20 \%$ rabbit serum for 30 min prior to a 2 -h incubation with primary antibody. FLOT1 monoclonal antibody was purchased from Cell Signaling Technology. The sections were washed twice with Tris-buffered saline, then incubated with biotinylated anti-rabbit antibody (Dako, Glostrup, Denmark). Analysis of the antibody reaction was performed with a streptavidin-biotin complex, and the results of immunohistochemistry were detected using a light microscope. FLOT1 immunostaining was semi-quantitatively evaluated for intensity (-, negative; +, weak; ++, moderate; and +++, strong).

RT-PCR and real-time PCR. Messenger RNA was extracted from normal bladder and TCC tissues using a Quick Prep mRNA Purification kit (GE Healthcare, Buckinghamshire, UK) according to the manufacturer's instructions. A FirstStrand cDNA Synthesis kit (Amersham Biosciences, Little Chalfont, UK) was used for reverse transcription. The PCR was carried out according to the manufacturer's instructions, and the PCR products were examined by agarose gel electrophoresis. Real-time PCR was performed using LC FastStart DNA Master SYBR-Green I (Roche, Carlsbad, CA, USA) and the PCR products were quantified with a LightCycler (Roche). The primers for FLOT1 were: forward, 5'-CCATCTCGTCAC TGGCATT-3' andreverse,5'-CGCCAACATCTCCTTGTTC-3'. The GAPDH (internal control) primer sequences were: forward, 5'-TCAAGAAGGTGGTGAAGCAGG-3' and reverse 5'-GTGGAGGAGTGGGTGTCG-3'.

Western blot analysis. Western blot analysis was carried out according to the manufacturer's instructions. Total protein was isolated and the protein concentration was measured, then SDS polyacrylamide gel electrophoresis was performed. Antibody to FLOT1 was purchased from Cell Signaling Technology, and an anti- $\beta$-actin monoclonal antibody (Abcam, Cambridge, UK) was used as an internal control. The immune complexes were determined using an ECL system (Amersham, Aylesbury, $\mathrm{UK})$.

RNAi and transfection. All siRNA oligonucleotide sequences were designed using the siDirect software. TCC cells were seeded in culture dishes with complete medium without antibiotics until the confluence reached 50-60\%. Then, TCC cells were transfected with siRNA oligonucleotides using Lipofectamine 2000 (Invitrogen, Carlsbad, CA, USA). After incubation for 2 days, FLOT1 protein expression was determined by western blot analysis. The cDNA coding sequence of FLOT1 was cloned by RT-PCR from normal human bladder tissue as a substrate, and the PCR products were subcloned into a pcDEF3 vector. Then, the 4 TCC cell lines were stably transfected with pcDEF3 vector containing full-length cDNA for FLOT1 using Lipofectamine 2000. The TCC monoclonal cell lines were selected with G418 and FLOT1 protein expression was confirmed by western blot analysis.

Proliferation analysis in vitro. The effect of FLOT1 on the proliferation of TCC cells was investigated using the WST-1 assay. Exponentially growing TCC cells $\left(4 \times 10^{3}\right)$ were harvested and seeded in 96-well microtiter plates. After continuous incubation for 1-3 days, $10 \mu$ l of WST-1 (Roche Diagnostics GmbH, Penzberg, Germany) was added to each well and the incubation was continued for an additional $2 \mathrm{~h}$. The absorbance of each well, representing the cell count, was detected with a microculture plate reader (Immunoreader; Japan Intermed Co., Ltd., Tokyo, Japan) at $450 \mathrm{~nm}$. 
Animal xenografts. Thirty BALB/C nude mice (4-5 weeks old) were randomly divided into the following 2 groups of 15 mice each: control group and FLOT1 group. A total of $3 \times 10^{7}$ TCC cells were injected into the back of each nude mouse, and all mice were continuously observed for 5 weeks; the volume of TCC tumors was recorded weekly. Five weeks later, all nude mice were sacrificed under deep anesthesia and the volume of each TCC tumor was measured.

\section{Adhesion, migration and invasion assay}

Adhesion assay. Twenty-four-well tissue culture plates were coated with $30 \mu \mathrm{g}$ of Matrigel/well and air dried for $50 \mathrm{~min}$. TCC cells $\left(5 \times 10^{4}\right)$ were suspended in RPMI-1640 containing $0.5 \%$ bovine serum albumin and dispensed into each well. The cells were incubated for $1 \mathrm{~h}$ and thrice-washed to remove the unattached cells. The TCC cells that remained attached to the bottom of the plate were stained with hematoxylin and counted with a microscope.

Migration assay. The migration assays were analyzed with a 24-well Transwell system (Poretics Corp., Livermore, CA, USA) containing $8 \mu \mathrm{m}$-pore polycarbonate membrane filters. TCC cells were seeded in the upper wells at a density of $1 \times 10^{4}$ cells/well, then incubated at $37^{\circ} \mathrm{C}$ for $24 \mathrm{~h}$. After incubation, the membranes were removed and fixed in $70 \%$ ethanol, then stained with hematoxylin for $30 \mathrm{~min}$. The upper side of the membrane was scraped to remove cells that had attached but not migrated, then the membrane was mounted onto a microscope slide. Chemotaxis was assessed in each well by counting the number of cells in 15 randomized fields under a microscope.

Invasion assay. TCC cell invasion was determined with a Transwell system containing a filter membrane with a diameter of $6.5 \mathrm{~mm}$ and pore size of $8 \mu \mathrm{m}$ (Corning, Inc., Corning, NY, USA). A thin gel layer was formed by adding Matrigel onto the filter, and a total of $1 \times 10^{5}$ cells was suspended in serum-free medium, then seeded into the upper chamber of the Transwell system. After a 24-h incubation, the cells on the upper filter were removed and the cells that penetrated to the lower filter were stained with hematoxylin. The cell count was performed under a microscope in 15 randomized fields.

Statistical analysis. SPSS (version 11.0) was used to perform statistical calculations. All experiments were carried out in triplicate and the results are expressed as the mean \pm standard deviation (SD). Statistical significance was determined using the Student's t-test, and a $\chi^{2}$ test was also used to determine the correlation between FLOT1 expression and the clinical characteristic of patients with TCC. A P-value $<0.05$ was considered to indicate a statistically significant difference.

\section{Results}

Patient clinical characteristics. There were 75 male and 81 female patients in the present study, with an age range of 38-85 years (mean, 59 years). Tumor size ranged from 0.5 to $14 \mathrm{~cm}$ (average, $1.9 \mathrm{~cm}$ ). One hundred and nine patients had primary TCCs and 47 patients had previously undergone transurethral resections or cystectomies. Among the TCC patients, 89,48 and 19 were classified as grades I, II and III according to the 2004 WHO classification, respectively. The clinical stage was reviewed based on the UICC 2009 TNM staging system; 51, 46, 37 and 22 were stages T1, T2, T3 and T4, respectively (Table I). The follow-up period was 22-64 months. Presenting symptoms included hematuria (124 patients), abdominal pain (32 patients), urinary discomfort (86 patients) and palpable masses (6 patients). Ten patients had metastatic disease at the time of diagnosis; anemia existed in 38 patients and 32 patients had $>2$ concomitant diseases (angina pectoris, diabetes mellitus and urolithiasis).

FLOT1 expression in TCCs by IHC. FLOT1 protein expression in human TCCs and normal urothelial tissues was detected by immunohistochemistry. The expression of FLOT1 TCC was significantly overexpressed compared to normal urothelial tissue (Fig. 1). Specifically, FLOT1 expression was detected in 145/156 TCCs (92.9\%); $44.1 \%$ of TCCs had moderate and strong FLOT1 expression. FLOT1 expression was only detected in 17/142 normal urothelial tissues (12\%); $23.5 \%$ of normal urothelial tissues had moderate and strong FLOT1 expression. The level of FLOT1 expression was significantly related to tumor size, pathologic grade, clinical stage and recurrence based on $\chi^{2}$ statistics $(\mathrm{P} \leq 0.05)$. In contrast, none of the other variables, such as gender and age, had a significant association with FLOT1 expression (Table I). These findings suggest that FLOT1 may be involved in tumorigenesis in human TCC.

FLOT1 expression in TCC by RT-PCR, real-time PCR and western blot analysis. FLOT1 expression in human TCCs and normal urothelial tissues was confirmed by RT-PCR, real-time PCR and western blot analysis. The relative level of FLOT1 expression was calculated as the ratio to the internal control. The results suggested that FLOT1 expression was upregulated significantly in TCCs compared to normal urothelial tissue, and the level of FLOT1 expression was similar to the levels detected by immunohistochemistry. Moreover, FLOT1 expression was significantly associated with tumor size, pathologic grade, clinical stage and recurrence based on t-tests $(\mathrm{P} \leq 0.05)$. The results of representative samples are shown in Fig. 2.

FLOT1 enhances the proliferation of TCC. An expression pcDEF3 vector containing the full-length cDNA for FLOT1 was stably transfected into 4 TCC cell lines (RT112, 253J, T24 and TCCSUP). In addition, FLOT1 expression was also decreased using RNAi. All transfected cell lines were confirmed by western blot analysis, and FLOT1 expression was significantly increased by the FLOT1 vector insert and decreased by siRNA (Fig. 3A). The effect of FLOT1 expression on the proliferation of TCC cells in vitro was evaluated by the WST-1 assay. TCC cells expressing high FLOT1 exhibited significantly increased proliferative ability compared to control cells; however, TCC cells with low FLOT1 expression had a lower proliferative ability (Fig. 3B). Moreover, similar results were also confirmed in vivo using an animal xenograft model with BALB/C nude mice (Fig. 3C). These findings suggested that FLOT1 may be involved in proliferation of human TCC. 
Table I. FLOT1 expression and characteristics of patients with TCC investigated by immunohistochemistry and real-time PCR.

\begin{tabular}{|c|c|c|c|c|c|c|c|c|}
\hline & \multirow[b]{2}{*}{$\mathrm{n}$} & \multirow[b]{2}{*}{ FLOT1 mRNA (mean \pm SD) } & \multirow[b]{2}{*}{ P-value } & \multicolumn{4}{|c|}{ FLOT1 protein } & \multirow[b]{2}{*}{ P-value } \\
\hline & & & & - & + & ++ & +++ & \\
\hline TCC & 156 & $2.31 \pm 0.35$ & & 15 & 77 & 42 & 22 & \\
\hline Normal bladder tissue & 142 & $0.71 \pm 0.22$ & $<0.05$ & 125 & 13 & 3 & 1 & $<0.05$ \\
\hline \multicolumn{9}{|l|}{ Gender } \\
\hline Male & 75 & $2.34 \pm 0.29$ & & 7 & 37 & 20 & 11 & \\
\hline Female & 81 & $2.29 \pm 0.27$ & $>0.05$ & 8 & 40 & 22 & 11 & $>0.05$ \\
\hline \multicolumn{9}{|l|}{ Age (years) } \\
\hline$<60$ & 95 & $2.28 \pm 0.26$ & & 9 & 45 & 27 & 14 & \\
\hline$\geq 60$ & 61 & $2.36 \pm 0.33$ & $>0.05$ & 6 & 32 & 15 & 8 & $>0.05$ \\
\hline \multicolumn{9}{|l|}{ Tumor size (cm) } \\
\hline$<3$ & 103 & $1.51 \pm 0.18$ & & 10 & 58 & 29 & 6 & \\
\hline$\geq 3$ & 53 & $3.86 \pm 0.55$ & $<0.05$ & 5 & 19 & 13 & 16 & $<0.05$ \\
\hline \multicolumn{9}{|l|}{ Histologic grade } \\
\hline I & 89 & $1.31 \pm 0.19$ & & 12 & 60 & 16 & 1 & \\
\hline II & 48 & $3.12 \pm 0.34$ & & 3 & 17 & 22 & 6 & \\
\hline III & 19 & $4.95 \pm 0.46$ & $<0.05$ & 0 & 0 & 4 & 15 & $<0.05$ \\
\hline \multicolumn{9}{|l|}{ Clinical stage } \\
\hline $\mathrm{T}_{1}-\mathrm{T}_{2}$ & 97 & $1.42 \pm 0.23$ & & 10 & 65 & 16 & 6 & \\
\hline $\mathrm{T}_{3}-\mathrm{T}_{4}$ & 59 & $3.77 \pm 0.36$ & $<0.05$ & 5 & 12 & 26 & 16 & $<0.05$ \\
\hline \multicolumn{9}{|l|}{ Primary or recurrence } \\
\hline Primary & 109 & $1.72 \pm 0.29$ & & 13 & 73 & 19 & 4 & \\
\hline Recurrence & 47 & $3.68 \pm 0.41$ & $<0.05$ & 2 & 4 & 23 & 18 & $<0.05$ \\
\hline
\end{tabular}
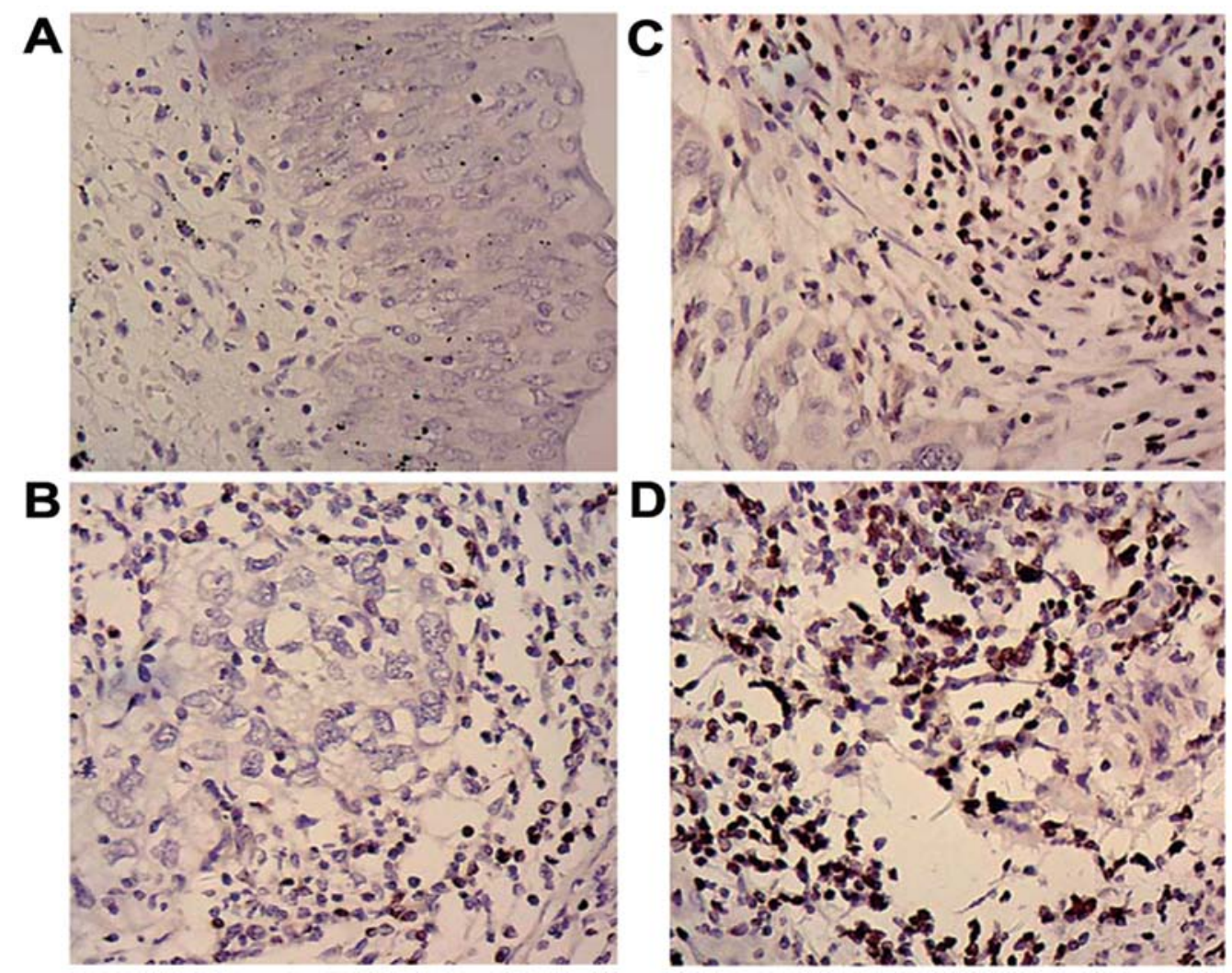

Figure 1. FLOT1 protein expression by immunohistochemical staining. (A) FLOT1 expression (-), (B) weakly positive FLOT1 expression (+), (C) moderately positive FLOT1 expression (++), and (D) strongly positive FLOT1 expression (+++). Original magnification, x200. 

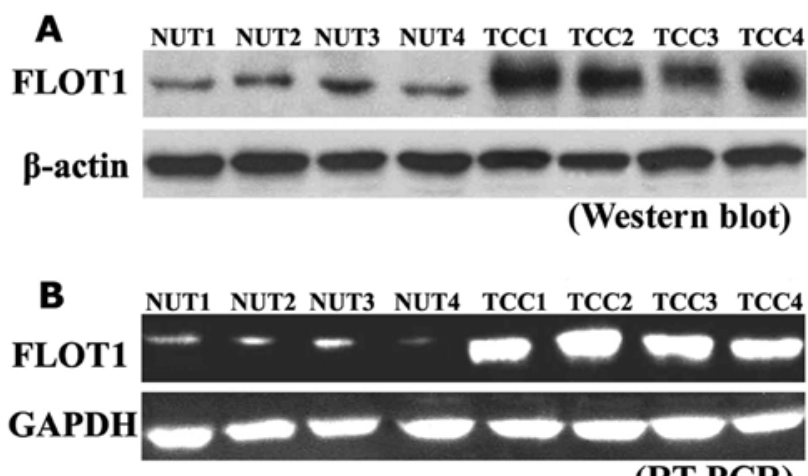

C

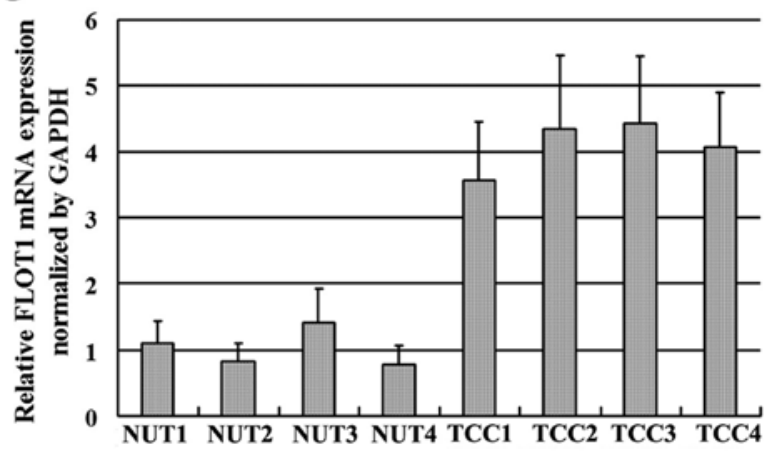

(Real-time PCR)

Figure 2. FLOT1 expression detected by (A) western blot analysis, (B) RT-PCR, and (C) real-time PCR. Results of representative samples are shown. All experiments were performed in triplicate and the error bar represents the SD. NUT, normal urothelial tissue.

FLOT1 enhances the adhesion, migration and invasion of TCC cells. The recurrence of TCC is related to spread by intraluminal seeding. Recurrence, a major cause of mortality in tumor patients, is a complex multistep process that includes cells adhesion, migration and invasion (25). The effects of FLOT1 on the adhesion, migration and invasion of TCC cells were evaluated in the present study. The expression of FLOT1 in 4 TCC cell lines was decreased by siRNA, and FLOT1 expression was increased by transfection with an expression pcDEF3 vector containing full-length cDNA. Protein expression was evaluated using western blot analysis (Fig. 3A). As shown in Fig. 3, TCC cells with high expression of FLOT1 had higher levels of adhesion (Fig. 4A), migration (Fig. 4B) and invasion (Fig. 4C) compared to untreated control cells. In contrast, TCC cells with low levels of FLOT1 due to siRNA treatment had significantly reduced adhesion (Fig. 4A), migration (Fig. 4B) and invasion (Fig. 4C) when compared to untreated control cells. These results suggest that FLOT1 can increase the adhesion, migration and invasion of TCC cells, and that FLOT1 may have an important role in TCC recurrence.

\section{Discussion}

Bladder TCC is the 9th most common carcinoma in humans, and the incidence is nearly 3 -fold higher in males than in females (26). TCC is a life-threatening disease due to the high rate of recurrence; radical cystectomy is the recommended
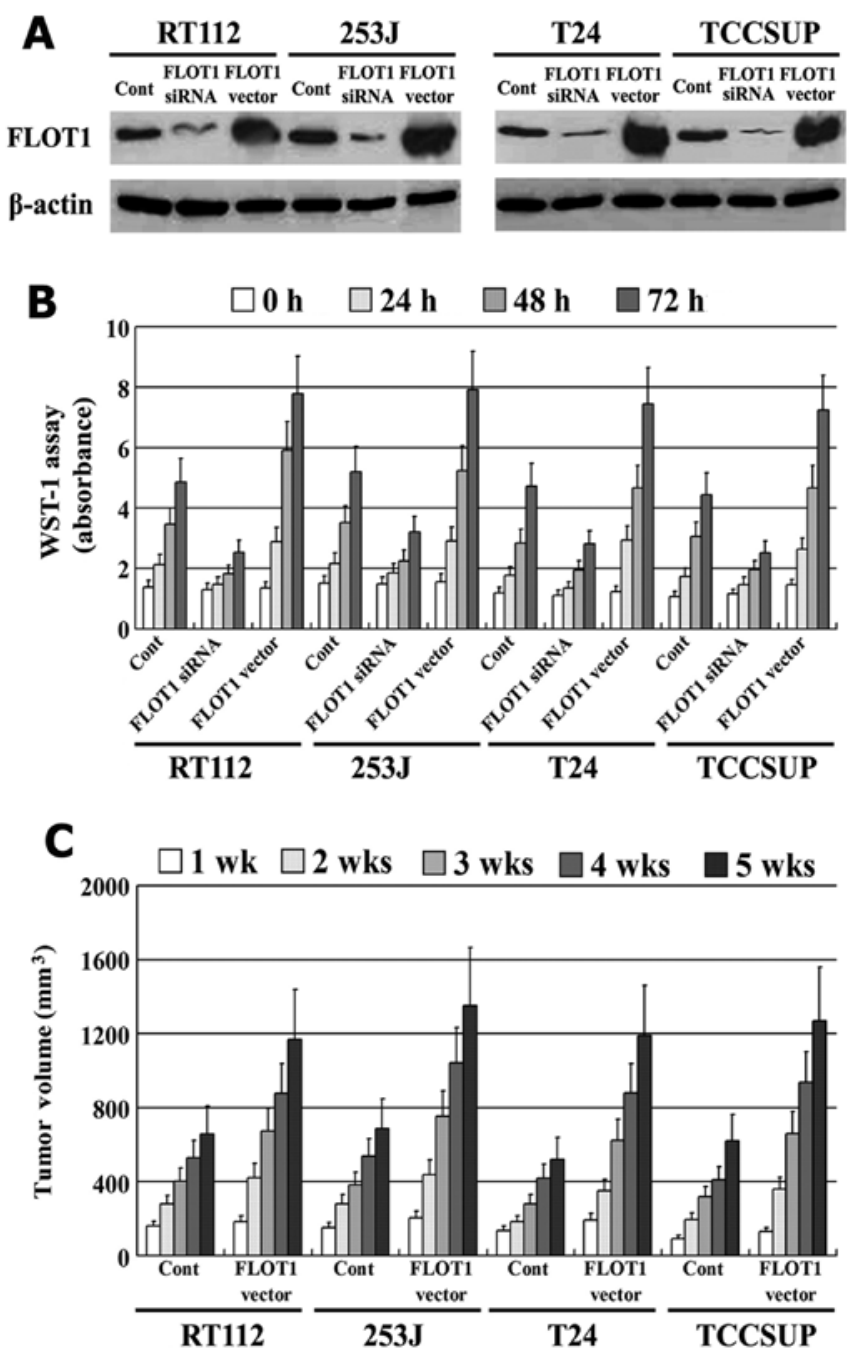

Figure 3. Effect of FLOT1 on the proliferation of TCC cells. pcDEF3 vector for FLOT1 was transfected stably into TCC cell lines. FLOT1 expression was also decreased using RNAi. (A) All transfections were confirmed by western blot analysis. The proliferative ability of TCC cells in vitro was evaluated by the WST-1 assay (B), and in vivo by an animal xenograft model with BALB/C nude mice (C).

treatment for muscle-invasive and recurrence-frequent disease. To prevent recurrence of TCC after transurethral resection, routine intravesical instillation with chemotherapeutic agents is usually performed (27). Although TCC is chemosensitive, no drugs are currently available to specifically block recurrence $(28,29)$. Thus, a novel mechanism and therapeutic innovation for treating patients with recurrent TCC is urgently required.

Recently, FLOT1 was thought to be an oncogene in tumorigenesis; high expression of FLOT1 has also been detected in various human cancers. A study reported that FLOT1 is involved in the regulation of ErbB2 activation in breast cancer, and decreasing FLOT1 expression significantly suppressed the proliferation of breast cancer cells in vitro and in vivo (30). Another study indicated that FLOT1 is associated with tumorigenesis and progression of hepatocellular carcinoma, and that FLOT1 may be useful as a prognostic marker in patients with hepatocellular carcinoma (31). Taken together, these findings demonstrated that FLOT1 plays an oncogenic role in human tumors. 


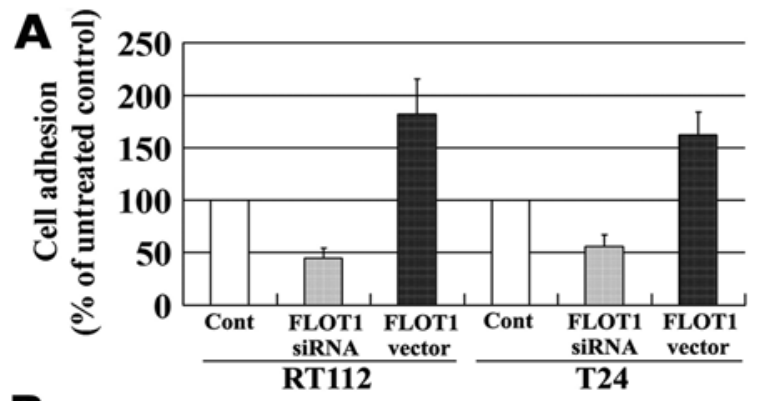

B
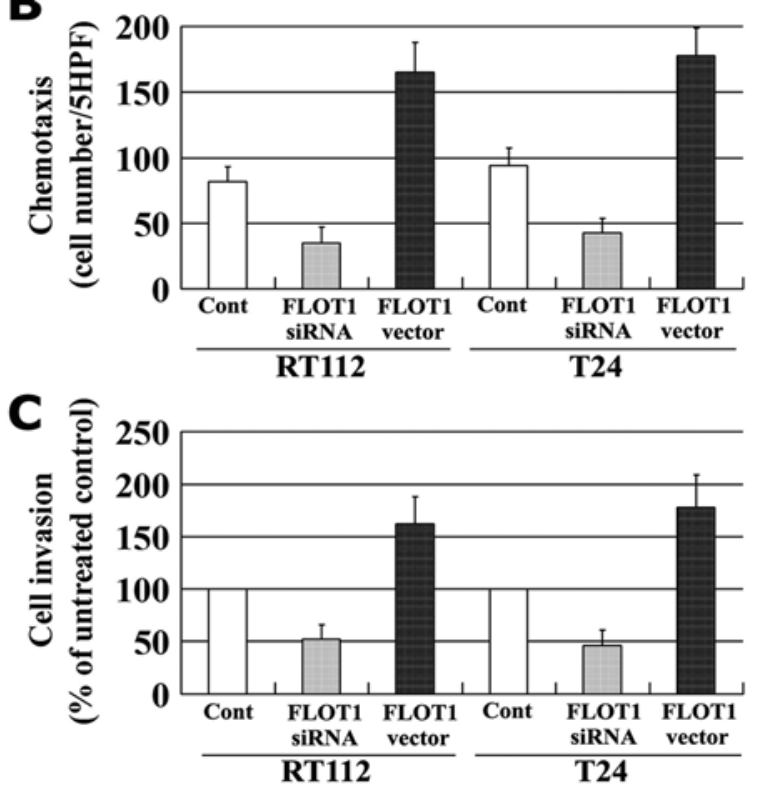

Figure 4. FLOT1 expression decreased by siRNA and increased by pcDEF3 vector transfection. FLOT1 increased the adhesion (A), migration (B), and invasion $(\mathrm{C})$ of TCC cells.

Although the potential oncogenic role of FLOT1 has been shown in prostate carcinoma (24), the function of FLOT1 in TCC remains unclear, and the effects and mechanisms of FLOT1 on TCC proliferation need to be further elucidated. This is the first study involving FLOT1 in human TCC. In the present study, we examined the expression of FLOT1 in human TCC and the results indicated that FLOT1 expression is significantly overexpressed in TCC compared to normal urothelial tissues, as shown by immunohistochemistry. Moreover, FLOT1 expression was significantly related to tumor size, pathologic grade, clinical stage and recurrence. FLOT1 expression in TCC was detected by real-time PCR, RT-PCR and western blot analysis, and the results were confirmed by immunohistochemistry. We further investigated the effect of FLOT1 on the proliferation of TCC cells, and showed that high expression of FLOT1 significantly increased the proliferation of TCC cells in vitro, and in vivo in a xenograft model with BALB/C nude mice. Collectively, these findings indicated that FLOT1 is a key gene in tumorigenesis and plays an important role in the progression of TCC.

Attempts have been made to predict recurrences in patients with TCCs; however, the mechanism underlying recurrence remains obscure (32-34). Since FLOT1 acts as an important gene in various biological behaviors, such as cell adhesion and invasion, a study suggested that FLOT1 can enhance cell invasion by increasing NF- $\kappa \mathrm{B}$ activation in esophageal squamous cell carcinoma cells (22). Therefore, we speculate that FLOT1 may participate in TCC recurrence. It has been reported that the recurrence of TCC is closely associated with spread by intraluminal seeding (35), and recurrence is a complex multistep process that includes adhesion, migration and invasion of tumor cells (36). Thus, the effects of FLOT1 on adhesion, migration and invasion of TCC cells were analyzed in the present study. Our data indicate that FLOT1 enhances the adhesion, migration and invasion potential of TCC. Moreover, FLOT1 is overexpressed in recurrent TCC compared to primary TCC based on real-time PCR and immunohistochemistry. These results suggest that FLOT1 prompts the adhesion, migration and invasion of TCC cells and leads to recurrent TCC.

In conclusion, our results indicated that FLOT1 expression is upregulated in human TCC and high expression of FLOT1 might lead to progression and recurrence of TCC. It is also possible that patients with high levels of FLOT1 expression may be vulnerable to the progression of TCC and FLOT1 may be a useful marker for recurrent TCC. Our findings suggest that FLOT1 plays an important role in the proliferation and recurrence of TCC and silencing FLOT1 expression may prove to be a novel therapeutic strategy. The detailed molecular mechanisms by which FLOT1 determines TCC characteristics warrant further investigation.

\section{References}

1. Ploeg M, Aben KK and Kiemeney LA: The present and future burden of urinary bladder cancer in the world. World J Urol 279: 289-293, 2009.

2. Jacobs BL, Lee CT and Montie JE: Bladder cancer in 2010: how far have we come? CA Cancer J Clin 60: 244-272, 2010.

3. Kumar V, Abbas AK and Fausto N: Pathologic Basis of Disease. 8th edition. Saunders Elsevier, Philadelephia, PA, pp976-980, 2010.

4. Shariat SF, Karakiewicz PI, Palapattu GS, et al: Outcomes of radical cystectomy for transitional cell carcinoma of the bladder: a contemporary series from the Bladder Cancer Research Consortium. J Urol 176: 2414-2422, 2006.

5. Morgan TM and Clark PE: Bladder cancer. Curr Opin Oncol 22: 242-249, 2010.

6. Patra SK: Dissecting lipid raft facilitated cell signaling pathways in cancer. Biochim Biophys Acta 1785: 182-206, 2008.

7. Patra SK and Bettuzzi S: Epigenetic DNA-methylation regulation of genes coding for lipid raft-associated components: A role for raft proteins in cell transformation and cancer progression (Review). Oncol Rep 17: 1279-1290, 2007.

8. Lingwood D and Simons K: Lipid rafts as a membrane-organizing principle. Science 327: 46-50, 2010.

9. Jacobson K, Mouritsen OG and Anderson RG: Lipid rafts: at a crossroad between cell biology and physics. Nat Cell Biol 9: 7-14, 2007.

10. Simons K and Toomre D: Lipid rafts and signal transduction. Nat Rev Mol Cell Biol 1: 31-39, 2000.

11. Mollinedo F, de la Iglesia-Vicente J, Gajate C, et al: Lipid rafttargeted therapy in multiple myeloma. Oncogene 29: 3748-3757, 2010.

12. Hitosugi T, Sato M, Sasaki K and Umezawa Y: Lipid raft specific knockdown of SRC family kinase activity inhibits cell adhesion and cell cycle progression of breast cancer cells. Cancer Res 67: 8139-8148, 2007.

13. Lang DM, Lommel S, Jung M, Ankerhold R and Petrausch B: Identification of reggie-1 and reggie-2 as plasmamembrane-associated proteins which cocluster with activated GPI-anchored cell adhesion molecules in non-caveolar micropatches in neurons. $J$ Neurobiol 37: 502-523, 1998.

14. Stuermer CA, Lang DM, Kirsch F, Wiechers M, Deininger SO and Plattner H: Glycosylphosphatidyl inositol-anchored proteins and fyn kinase assemble in noncaveolar plasma membrane microdomains defined by reggie-1 and -2 . Mol Biol Cell 12: 3031-3045, 2001 
15. Schulte T, Paschke KA, Laessing U, Lottspeich $F$ and Stuermer CA: Reggie-1 and reggie-2, two cell surface proteins expressed by retinal ganglion cells during axon regeneration. Development 124: 577-587, 1997.

16. Banning A, Tomasovic A and Tikkanen R: Functional aspects of membrane association of reggie/flotillin proteins. Curr Protein Pept Sci 12: 725-735, 2011.

17. Riento K, Frick M, Schafer I and Nichols BJ: Endocytosis of flotillin-1 and flotillin-2 is regulated by Fyn kinase. J Cell Sci 122: 912-918, 2009.

18. Baumann CA, Ribon V, Kanzaki M, et al: CAP defines a second signaling pathway required for insulin-stimulated glucose transport. Nature 407: 202-207, 2000.

19. Lin C, Wu Z, Lin X, et al: Knockdown of FLOT1 impairs cell proliferation and tumorigenicity in breast cancer through upregulation of FOXO3a. Clin Cancer Res 17: 3089-3099, 2011.

20. Hazarika P, McCarty MF, Prieto VG, et al: Up-regulation of Flotillin-2 is associated with melanoma progression and modulates expression of the thrombin receptor protease activated receptor 1. Cancer Res 64: 7361-7369, 2004.

21. Thorn CC, Freeman TC, Scott N, Guillou PJ and Jayne DG: Laser microdissection expression profiling of marginal edges of colorectal tumours reveals evidence of increased lactate metabolism in the aggressive phenotype. Gut 58: 404-412, 2009.

22. Song L, Gong H, Lin C, et al: Flotillin-1 promotes tumor necrosis factor- $\alpha$ receptor signaling and activation of NF- $\kappa \mathrm{B}$ in esophageal squamous cell carcinoma cells. Gastroenterology 143: 995-1005.e12, 2012

23. Santamaria A, Castellanos E, Gomez V, et al: PTOV1 enables the nuclear translocation and mitogenic activity of flotillin-1, a major protein of lipid rafts. Mol Cell Biol 25: 1900-1911, 2005.

24. Gomez V, Sese M, Santamara A, et al: Regulation of aurora B kinase by the lipid raft protein flotillin-1. J Biol Chem 285 : 20683-20690, 2010.

25. Sternlicht MD and Werb Z: How matrix metalloproteinases regulate cell behavior. Annu Res Cell Dev Biol 17: 465-516, 2001.

26. Lerner SP: Bladder cancer clinical trials. Urol Oncol 23: 275-279, 2005.

27. Mitsumori K, Tsuchiya N, Habuchi T, et al: Early and largedose intravesical instillation of epirubicin to prevent superficial bladder carcinoma recurrence after transurethral resection. BJU Int 94: 317-321, 2004.
28. Brausi M, Collette L and Kurth K: Variability in the recurrence rate at first follow up cystoscopy after TUR in stage Ta T1 transitional cell carcinoma of the bladder: a combined analysis of seven EORTC studies. Eur Urol 41: 523-531, 2002.

29. Sylvester RJ, Oosterlinck W and Van der Meijden AP: A single immediate postoperative instillation of chemotherapy decreases the risk of recurrence in patients with stage TaT1 bladder cancer: a meta analysis of published results of randomized clinical trials. J Urol 171: 2186-2190, 2004.

30. Pust S, Klokk TI, Musa N, et al: Flotillins as regulators of ErbB2 levels in breast cancer. Oncogene 32: 3443-3451, 2013.

31. Zhang SH, Wang CJ, Shi L, et al: High expression of FLOT1 is associated with progression and poor prognosis in hepatocellular carcinoma. PLoS One 8: e64709, 2013.

32. Ajili F, Darouiche A, Chebil M and Boubaker S: The efficiency of the EORTC scoring system for the prediction of recurrence and progression of non-muscle-invasive bladder cancer treated by bacillus calmette-guerin immunotherapy. Ultrastruct Pathol 37: 249-253, 2013.

33. Xu T, Zhu Z, Zhang X, et al: Predicting recurrence and progression in chinese patients with nonmuscle-invasive bladder cancer using EORTC and CUETO scoring models. Urology 82: 387-393, 2013.

34. Mares J, Szakacsova M, Soukup V, et al: Prediction of recurrence in low and intermediate risk non-muscle invasive bladder cancer by real-time quantitative PCR analysis: cDNA microarray results. Neoplasma 60: 295-301, 2013.

35. Hafner C, Knuechel R, Zanardo L, et al: Evidence for oligoclonality and tumor spread by intraluminal seeding in multifocal urothelial carcinomas of the upper and lower urinary tract. Oncogene 20: 4910-4915, 2001.

36. Shang D, Liu Y, Yang P, et al: TGFBI-promoted adhesion, migration and invasion of human renal cell carcinoma depends on inactivation of von Hippel-Lindau tumor suppressor. Urology 79: 966e1-966e7, 2012. 